\title{
Bistable scattering in graphene-coated dielectric nanowires
}

\author{
Rujiang $\mathrm{Li}^{1,2,3}$, Huaping Wang ${ }^{4}$, Bin Zheng ${ }^{1,2,3}$, Shahram Dehdashti ${ }^{1,2,3}$, Erping Li $^{2}$, and Hongsheng Chen ${ }^{1,2,3}$ * \\ ${ }^{1}$ State Key Laboratory of Modern Optical Instrumentation, Zhejiang University, Hangzhou 310027, China \\ ${ }^{2}$ College of Information Science and Electronic Engineering, Zhejiang University, Hangzhou 310027, China \\ ${ }^{3}$ The Electromagnetics Academy of Zhejiang University, \\ Zhejiang University, Hangzhou 310027, China and \\ ${ }^{4}$ Institute of Marine Electronics Engineering, Zhejiang University, Hangzhou 310058, China
}

\begin{abstract}
In nonlinear plasmonics, the switching threshold of optical bistability is limited by the weak nonlinear responses from the conventional Kerr dielectric media. Considering the giant nonlinear susceptibility of graphene, here we develop a nonlinear scattering model under the mean field approximation and study the bistable scattering in graphene-coated dielectric nanowires based on the semi-analytical solutions. We find that the switching intensities of bistable scattering can be smaller than $1 \mathrm{MW} / \mathrm{cm}^{2}$ at the working frequency. To further decrease the switching intensities, we show that the most important factor that restricts the bistable scattering is the relaxation time of graphene, rather than the chemical potential and the permittivity of the dielectric nanowire. Our work not only reveals some general characteristics of graphene-based bistable scattering, but also provides a guidance to further applications of optical bistability in the high speed all-optical signal processing.
\end{abstract}

\section{INTRODUCTION}

An optical device is said to be bistable if it has two stable output states under a same input state [1, 2]. The output can be switched between the two stable transmission states, depending upon the history of the input signal. Optical bistability is a nonlinear phenomenon and it has promising applications in all-optical switching [35], all-optical logic gates 66 9], optical transistors [10 12, and optical memory devices [13 16]. To produce a large nonlinear effect and decrease the switching threshold of optical bistability, nonlinear media with high Kerr coefficients are used and sophisticated structures that can enhance the local field intensities are designed.

Plasmonics is a rapidly growing field which provides an efficient way to enhance the local electromagnetic fields at subwavelength scales [17, 18. When nonlinear dielectric media are incorporated into the plasmonic structures, large nonlinear effects arise due to the enhanced local fields [19]. Various kinds of nonlinear plasmonic structures are designed to show the optical bistability [20 27]. However, the commonly used conventional Kerr dielectric materials can only display very weak nonlinear responses, which restrict further applications of optical bistability in the high speed all-optical signal processing.

As a newly discovered two dimensional material, graphene shows intriguing nonlinear characteristics in plasmonics 28. Compared with the conventional Kerr dielectric media, graphene has a giant nonlinear susceptibility [29]. The use of graphene along with the enhancement of local plasmonic fields can produce an extremely large nonlinear effect and consequently decrease the switching threshold of optical bistability. Recently, some graphene based plasmonic structures have been de-

*Electronic address: hansomchen@zju.edu.cn signed theoretically or realized experimentally to study the optical bistability, e.g. sandwiched structures $30-$ 32, a modified Kretschmann-Raether configuration 33, an Otto configuration [34, graphene nanoribbons [35], and graphene nanobubbles 36 .

Compared with those sophisticated structures, graphene-coated dielectric nanowires and nanospheres are two kinds of simple structures that are more interested to investigate. Such graphene based cylindrical and spherical structures always demonstrate some typical optical phenomena and can be very useful in lots of applications, such as cloaking [37, 38, superscattering 39 41, linear and nonlinear waveguiding [42 45], localized plasmons [46, and second harmonic generations 47. The analytical or semi-analytical solutions calculated from these simple models are not only useful to reveal some general characteristics, but also provide a guidance to further structure design and optimization [48].

In this paper, as a demonstration purpose, we study the bistable scattering in graphene-coated dielectric nanowires. A nonlinear scattering model is developed based on the Mie scattering theory. Under the mean field approximation, this model is solved semi-analytically in both the lossless and lossy cases. At the working frequency, the switching-up and switching-down intensities of bistable scattering are smaller than $1 \mathrm{MW} / \mathrm{cm}^{2}$. We show that the most important factor that restricts the further decrease of switching intensities is the relaxation time of graphene. Our work reveals some general characteristics of graphene-based bistable scattering, and provides a theoretical guidance to further structure design and optimization. 


\section{SURFACE CONDUCTIVITY OF GRAPHENE}

The optical response of graphene is contributed by both the intraband and interband transitions. For a doped monolayer graphene, the intraband transition dominates in the classical frequency range $\hbar \omega \leq \mu_{c} 28$. If the graphene monolayer is placed on the $x y$ plane and a time-dependent electric field of the form $\mathbf{E}(t)=$ $\hat{x}\left[E_{x}(\omega) e^{-i \omega t}+\right.$ c.c. $]+\hat{y}\left[E_{y}(\omega) e^{-i \omega t}+\right.$ c.c. $]$ is applied, the surface electric current is

$$
\mathbf{J}(t)=\hat{x}\left[j_{x}(\omega) e^{-i \omega t}+\text { c.c. }\right]+\hat{y}\left[j_{y}(\omega) e^{-i \omega t}+\text { c.c. }\right],
$$

where

$$
\begin{aligned}
j_{i}(\omega)= & \sigma_{i i}^{(\omega)} E_{i}+\left(\sigma_{i i j j}^{(3, \omega)}+\sigma_{i j j i}^{(3, \omega)}\right) E_{i}\left|E_{j}\right|^{2} \\
& +\sigma_{i j i j}^{(3, \omega)} E_{i}^{*} E_{j}^{2}+\sigma_{i i i i}^{(3, \omega)}\left|E_{i}\right|^{2} E_{i}
\end{aligned}
$$

is the surface electric current in $i$ direction in the frequency domain,

$$
\sigma_{i i}^{(\omega)}=i \frac{e^{2} \mu_{c}}{\pi \hbar^{2}(\omega+i / \tau)}
$$

is the tensor element of the linear surface conductivity,

$$
\sigma_{i i j j}^{(3, \omega)}=\sigma_{i j j i}^{(3, \omega)}=\sigma_{i j i j}^{(3, \omega)}=\sigma_{i i i i}^{(3, \omega)} / 3=-i \frac{3 e^{4} v_{F}^{2}}{8 \pi \mu_{c} \hbar^{2} \omega^{3}}
$$

is the tensor element of the nonlinear surface conductivity, $\mu_{c}$ is the chemical potential, $\tau$ is the relaxation time, $i, j=x, y$, and c.c. denotes the complex conjugate 30, 45. If the applied electric field components satisfy $E_{x} E_{y}^{*}=E_{x}^{*} E_{y}$, the surface electric current in $i$ direction in the frequency domain reduces to

$$
j_{i}(\omega)=\sigma_{g} E_{i}(\omega)
$$

where

$$
\sigma_{g}=\sigma_{g}^{(1)}+\sigma_{g}^{(3)}\left|\mathbf{E}_{\|}\right|^{2}
$$

is the surface conductivity of graphene,

$$
\sigma_{g}^{(1)}=\sigma_{i i}^{(\omega)}=i \frac{e^{2} \mu_{c}}{\pi \hbar^{2}(\omega+i / \tau)}
$$

is the linear part,

$$
\sigma_{g}^{(3)}=\sigma_{i i i i}^{(3, \omega)}=-i \frac{9 e^{4} v_{F}^{2}}{8 \pi \mu_{c} \hbar^{2} \omega^{3}},
$$

is the coefficient of the nonlinear part, and $\mathbf{E}_{\|}=\hat{x} E_{x}+$ $\hat{y} E_{y}$ is the electric field that is parallel to the graphene surface. The above calculation results are valid for $\omega \tau \gg$ 1 [45, where the relaxation time of graphene $\tau$ which describes the dissipation loss usually ranges from $0.01 \mathrm{ps}$ to $1 \mathrm{ps} 28$. dielectric nanowire graphene

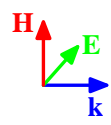

FIG. 1: Schematic of the infinitely long graphene-coated dielectric nanowire. A TM-polarized plane wave with the field components $H_{z}, E_{r}$, and $E_{\theta}$ is incident normally from air onto the structure. The relative permittivity and permeability of the dielectric nanowire are $\varepsilon_{r}=2.7$ and $\mu_{r}=1$, respectively, and the radius of the nanowire is $R=200 \mathrm{~nm}$.

\section{NONLINEAR SCATTERING MODEL}

As shown in Fig. 1, a TM-polarized plane wave with the field components $H_{z}, E_{r}$, and $E_{\theta}$ is incident normally from air onto an infinitely long graphene-coated dielectric nanowire. The incident magnetic field is $\mathbf{H}(t)=$ $\hat{z}\left(H_{0} e^{i k_{0} x-i \omega t}+\right.$ c.c. $) / 2$, where $k_{0}$ is the wavenumber in free space, $\omega=2 \pi f$ is the angular frequency. The relative permittivity of the lossless dielectric nanowire is assumed as $\varepsilon_{r}=2.7$, and the relative permeability is $\mu_{r}=1$. The radius of the dielectric nanowire is $R=200 \mathrm{~nm}$.

Since the thickness of the graphene coating is extremely small compared with its radius, the graphene coating can be well characterized as a two dimensional homogenized conducting film, where the microscopic details are neglected [46], as shown in Fig. 1. For this structure, only the azimuthal component of the electric field $E_{\theta}$ contributes to the nonlinear surface conductivity of graphene, and the surface electric current along the graphene coating is

$$
j_{\theta}(\omega)=\left(\sigma_{g}^{(1)}+\sigma_{g}^{(3)}\left|E_{\theta}\right|^{2}\right) E_{\theta}(\omega)
$$

where $\mathbf{J}(t)=\hat{\theta}\left(j_{\theta} e^{-i \omega t}+\right.$ c.c. $), \mathbf{E}(t)=\hat{\theta}\left(E_{\theta} e^{-i \omega t}+\right.$ c.c. $)$, and the values of $\sigma_{g}^{(1)}$ and $\sigma_{g}^{(3)}$ are calculated from Eqs. (7)-(8), respectively.

From Mie scattering theory, the electromagnetic fields inside and outside the dielectric nanowire can be expressed explicitly [39, 49]. According to the boundary condition of the tangential electric field $E_{\theta}$ at $r=R$, we have

$$
a_{n}\left[J_{n}^{\prime}\left(k_{0} R\right)+s_{n} H_{n}^{(1) \prime}\left(k_{0} R\right)\right]=\frac{1}{\sqrt{\varepsilon_{r}}} b_{n} J_{n}^{\prime}(k R),
$$

where $a_{n}=\delta_{n} i^{n}\left(\delta_{n}=1\right.$ for $n=0$ and $\delta_{n}=2$ for $\left.n \neq 0\right)$, $k=k_{0} \sqrt{\varepsilon_{r}}$ is the wavenumber in the dielectric nanowire, $s_{n}$ is the scattering coefficient, and $J_{n}$ and $H_{n}^{(1)}$ are the $n$-th order Bessel function of the first kind and Hankel function of the first kind, respectively. Then according 
to the another boundary condition from $H_{z}$, we have

$$
\begin{aligned}
& \sum_{n=0}^{\infty} a_{n}\left[J_{n}\left(k_{0} R\right)+s_{n} H_{n}^{(1)}\left(k_{0} R\right)\right] \cos (n \theta) \\
= & \sum_{n=0}^{\infty} b_{n}\left[J_{n}(k R)+i \frac{\sigma_{g} \eta_{0}}{\sqrt{\varepsilon_{r}}} J_{n}^{\prime}(k R)\right] \cos (n \theta),
\end{aligned}
$$

where

$$
\sigma_{g}=\sigma_{g}^{(1)}+\frac{\sigma_{g}^{(3)} \eta_{0}^{2} H_{0}^{2}}{4 \varepsilon_{r}}\left|\sum_{m=0}^{\infty} b_{m} J_{m}^{\prime}(k R) \cos (m \theta)\right|^{2}
$$

From this general boundary condition, we can see that scattering terms with different orders are coupled via the nonlinear surface conductivity of graphene. Since a scattering term with a given order contributes to the scattering terms with other orders, the total surface conductivity of graphene is inhomogeneous along the azimuthal direction.

In Eq. (9), the nonlinear part is usually small compared with the linear part. We can use the mean field approximation to simplify the nonlinear scattering model, where the field intensity at the circumference of the graphene coating is represented by the average value [23, 26]. From Eq. 12, we have

$$
\begin{aligned}
\sigma_{g}= & \sigma_{g}^{(1)}+\frac{\sigma_{g}^{(3)} \eta_{0}^{2} H_{0}^{2}}{8 \pi \varepsilon_{r}} \sum_{m=0}^{\infty} \sum_{m^{\prime}=0}^{\infty} b_{m} b_{m^{\prime}}^{*} J_{m}^{\prime}(k R) J_{m^{\prime}}^{\prime *}(k R) \\
& \times \int_{0}^{2 \pi} \cos (m \theta) \cos \left(m^{\prime} \theta\right) d \theta
\end{aligned}
$$

which simplifies to

$$
\begin{aligned}
\sigma_{g}= & \sigma_{g}^{(1)}+\frac{\sigma_{g}^{(3)} \eta_{0}^{2} H_{0}^{2}}{4 \varepsilon_{r}}\left|b_{0}\right|^{2} J_{0}^{\prime 2}(k R) \\
& +\frac{\sigma_{g}^{(3)} \eta_{0}^{2} H_{0}^{2}}{8 \varepsilon_{r}} \sum_{m=1}^{\infty}\left|b_{m}\right|^{2} J_{m}^{\prime 2}(k R) .
\end{aligned}
$$

Thus, under the mean field approximation, the surface conductivity of graphene is independent on the $\theta$. But scattering terms with different orders are still coupled.

If the radius of the dielectric nanowire is far smaller than the wavelength, the total scattering is dominated by the $m=1$ term [39] (Supplement 1, Section 1). The surface conductivity of graphene reduces to

$$
\sigma_{g}=\sigma_{g}^{(1)}+\frac{\sigma_{g}^{(3)} \eta_{0}^{2} H_{0}^{2}}{8 \varepsilon_{r}}\left|b_{1}\right|^{2} J_{1}^{\prime 2}(k R) .
$$

In this case, the surface conductivity of graphene only depends on the first order scattering term, and the other scattering terms are coupled with the first order scattering term via the surface conductivity. However, the nonlinear part of the surface conductivity is usually small compared with the linear part, and the contribution from the first order scattering term to the other terms are small. The total scattering can be simplified further by neglecting the weak coupling between the terms. For $n=1$, Eq. 111 reduces to

$$
\begin{aligned}
& \sqrt{\varepsilon_{r}} a_{1}\left[J_{1}\left(k_{0} R\right)+s_{1} H_{1}^{(1)}\left(k_{0} R\right)\right] \\
& -b_{1}\left[\sqrt{\varepsilon_{r}} J_{1}(k R)+i \sigma_{g}^{(1)} \eta_{0} J_{1}^{\prime}(k R)\right] \\
= & \alpha b_{1}\left|b_{1}\right|^{2} J_{1}^{\prime 3}(k R),
\end{aligned}
$$

where $\alpha=i \sigma_{g}^{(3)} \eta_{0}^{3} H_{0}^{2} /\left(8 \varepsilon_{r}\right)$. This is the boundary condition for $H_{z}$ under the mean field approximation.

From the two boundary conditions shown by Eqs. 10 and (16), we obtain a cubic nonlinear equation for the first order scattering coefficient $s_{1}$ (Supplement 1, Section 2). In order to solve this equation, for simplicity, we can use the approximations of $k_{0} R \ll 1$ and $k R \ll 1$ since the wavelengths are much larger than the radius of the dielectric nanowire. Using the asymptotic expansions of the Bessel function and Hankel function [50], the nonlinear equation reduces to

$$
\begin{aligned}
& c^{(1)}+c_{1}^{(1)} s_{1}+c_{1 *}^{(1)} s_{1}^{*}+c_{11}^{(1)} s_{1}^{2}+c_{11^{*}}^{(1)}\left|s_{1}\right|^{2} \\
& +c_{11^{*} 1}^{(1)}\left|s_{1}\right|^{2} s_{1} \\
= & 0
\end{aligned}
$$

where the coefficients are

$$
\begin{aligned}
& c^{(1)}=\frac{1}{4} \alpha \varepsilon_{r}+\frac{1}{4}\left[\left(\varepsilon_{r}-1\right) k_{0} R+i \sigma_{g}^{(1)} \eta_{0}\right], \\
& c_{1}^{(1)}=i \frac{2 \alpha \varepsilon_{r}}{\pi k_{0}^{2} R^{2}}+i \frac{1}{\pi k_{0}^{2} R^{2}}\left[\left(\varepsilon_{r}+1\right) k_{0} R+i \sigma_{g}^{(1)} \eta_{0}\right],
\end{aligned}
$$

$$
\begin{aligned}
c_{1^{*}}^{(1)} & =-i \frac{\alpha \varepsilon_{r}}{\pi k_{0}^{2} R^{2}}, \\
c_{11}^{(1)} & =-\frac{4 \alpha \varepsilon_{r}}{\pi^{2} k_{0}^{4} R^{4}}, \\
c_{11^{*}}^{(1)} & =\frac{8 \alpha \varepsilon_{r}}{\pi^{2} k_{0}^{4} R^{4}}, \\
c_{11^{*} 1}^{(1)} & =i \frac{16 \alpha \varepsilon_{r}}{\pi^{3} k_{0}^{6} R^{6}} .
\end{aligned}
$$

Specially, if the nonlinear surface conductivity of graphene is neglected, namely in the limit of $H_{0}=0$, the scattering coefficient reduces to

$$
s_{1}=i \frac{\pi k_{0}^{2} R^{2}}{4} \frac{\left(\varepsilon_{r}-1\right) k_{0} R+i \sigma_{g}^{(1)} \eta_{0}}{\left(\varepsilon_{r}+1\right) k_{0} R+i \sigma_{g}^{(1)} \eta_{0}} .
$$

After calculating the scattering coefficient, the normalized scattering cross section section (NSCS) can be evaluated as

$$
\mathrm{NSCS}=2\left|s_{1}\right|^{2}
$$

where only the first order scattering term is considered. 


\section{BISTABLE SCATTERING}

In the following, we study the bistable scattering in graphene-coated dielectric nanowires based on the nonlinear scattering model. As an example, the chemical potential of graphene is chosen as $\mu_{c}=0.35 \mathrm{eV}$.

According to Eqs. (6)-(8), the surface conductivity of graphene has two parts, where the linear part is a complex number and the nonlinear part is a pure imaginary number. From Eqs. (18)-(23), only the two coefficients $c^{(1)}$ and $c_{1}^{(1)}$ are complex, while the other coefficients are all real. Thus, Eq. (17) is a nonlinear equation with complex coefficients and it only has complex solutions generally. Specially, if the relaxation time of graphene is infinite, namely if there is no dissipation loss, the linear surface conductivity in Eq. (7) only has the imaginary part and Eq. (17) has pure imaginary solutions.

\section{A. Lossless Case}

First we consider the lossless case, where the relaxation time of graphene is infinite. Since $s_{1}$ reduces to a pure imaginary number, we define $s_{1}=i s_{01}$. Then Eq. (17) reduces to

$$
c^{(1)}+c_{1}^{(1)} s_{01}+c_{11}^{(1)} s_{01}^{2}+c_{111}^{(1)} s_{01}^{3}=0,
$$

where the coefficients are

$$
\begin{aligned}
c^{(1)} & =-\frac{\sigma_{g, i}^{(3)} \eta_{0}^{2} I_{0}}{8}+\frac{1}{2}\left[\left(\varepsilon_{r}-1\right) k_{0} R-\sigma_{g, i}^{(1)} \eta_{0}\right] \\
c_{1}^{(1)} & =\frac{3 \sigma_{g, i}^{(3)} \eta_{0}^{2} I_{0}}{2 \pi k_{0}^{2} R^{2}}-\frac{2}{\pi k_{0}^{2} R^{2}}\left[\left(\varepsilon_{r}+1\right) k_{0} R-\sigma_{g, i}^{(1)} \eta_{0}\right] \\
c_{11}^{(1)} & =-\frac{6 \sigma_{g, i}^{(3)} \eta_{0}^{2} I_{0}}{\pi^{2} k_{0}^{4} R^{4}} \\
c_{111}^{(1)} & =\frac{8 \sigma_{g, i}^{(3)} \eta_{0}^{2} I_{0}}{\pi^{3} k_{0}^{6} R^{6}}
\end{aligned}
$$

the linear and nonlinear surface conductivities are

$$
\begin{aligned}
\sigma_{g, i}^{(1)} & =\operatorname{Im} \sigma_{g}^{(1)}=\frac{e^{2} \mu_{c}}{\pi \hbar^{2} \omega}, \\
\sigma_{g, i}^{(3)} & =\operatorname{Im} \sigma_{g}^{(3)}=-\frac{9 e^{4} v_{F}^{2}}{8 \pi \mu_{c} \hbar^{2} \omega^{3}},
\end{aligned}
$$

and $I_{0}=\eta_{0} H_{0}^{2} / 2$ is the incident field intensity. Specially, in the limit of $I_{0}=0$, the nonlinear equation reduces to the linear case, and the scattering coefficient is

$$
s_{1}=i s_{01}=i \frac{\pi k_{0}^{2} R^{2}}{4} \frac{\left(\varepsilon_{r}-1\right) k_{0} R-\sigma_{g, i}^{(1)} \eta_{0}}{\left(\varepsilon_{r}+1\right) k_{0} R-\sigma_{g, i}^{(1)} \eta_{0}} .
$$

The scattering coefficient in the linear case can be directly calculated from Eq. (33). Figs. 2(a)-(b) show
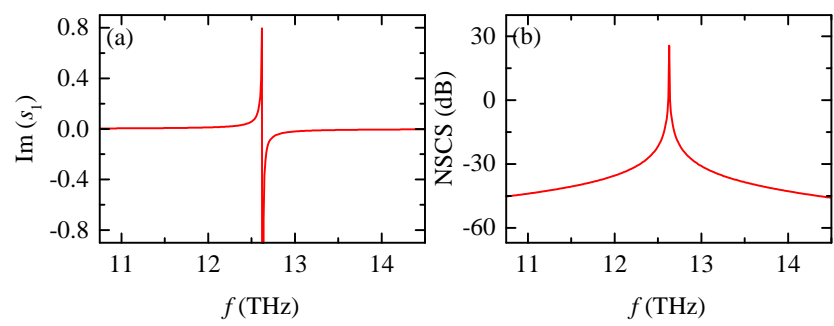

FIG. 2: (a) The scattering coefficients and (b) NSCSs at different frequencies, where $I_{0}=0, \mu_{c}=0.35 \mathrm{eV}$, and $\tau=\infty$.

the scattering coefficients and NSCSs at different frequencies, where $I_{0}=0, \mu_{c}=0.35 \mathrm{eV}$, and $\tau=\infty$. The NSCS exhibits the superscattering at $f_{0}=12.63$ THz, where $\left(\varepsilon_{r}+1\right) k_{0} R=\sigma_{g, i}^{(1)} \eta_{0}$, and the scattering coefficient shows a phase change of $\pi$ at the superscattering frequency [39]. According to Fig. 2(b), in order to enhance the local field intensity along the graphene coating in the nonlinear case, the working frequency for bistable scattering should be near to the superscattering frequency.

Eq. (26) can be solved analytically 50, where only the real solutions are physically acceptable. When $I_{0} \neq 0$, we define

$$
\begin{aligned}
& p=\frac{c_{1}^{(1)}}{c_{111}^{(1)}}-\frac{c_{11}^{(1) 2}}{3 c_{111}^{(1) 2}}, \\
& q=\frac{c^{(1)}}{c_{111}^{(1)}}+\frac{2 c_{11}^{(1) 3}}{27 c_{111}^{(1) 3}}-\frac{c_{11}^{(1)} c_{1}^{(1)}}{3 c_{111}^{(1) 2}}
\end{aligned}
$$

then the three solutions can be written as

$$
\begin{aligned}
& s_{01,1}=A^{1 / 3}+B^{1 / 3}+\Gamma, \\
& s_{01,2}=\omega_{+} A^{1 / 3}+\omega_{-} B^{1 / 3}+\Gamma, \\
& s_{01,3}=\omega_{-} A^{1 / 3}+\omega_{+} B^{1 / 3}+\Gamma,
\end{aligned}
$$

where $A=-q / 2+\sqrt{\Delta}, B=-q / 2-\sqrt{\Delta}, \Gamma=-b /(3 a)$, $\omega_{ \pm}=(-1 \pm \sqrt{3} i) / 2$, and

$$
\Delta=\left(\frac{q}{2}\right)^{2}+\left(\frac{p}{3}\right)^{3}
$$

If $\Delta>0, A$ and $B$ are both real numbers with $A \neq B$. The three solutions are

$$
\begin{aligned}
& s_{01,1}=A^{1 / 3}+B^{1 / 3}+\Gamma, \\
& s_{01,2}=-\frac{1}{2}\left(A^{1 / 3}+B^{1 / 3}\right)+\frac{\sqrt{3}}{2} i\left(A^{1 / 3}-B^{1 / 3}\right)+\Gamma, \\
& s_{01,3}=-\frac{1}{2}\left(A^{1 / 3}+B^{1 / 3}\right)-\frac{\sqrt{3}}{2} i\left(A^{1 / 3}-B^{1 / 3}\right)+\Gamma,
\end{aligned}
$$

namely, only $s_{01,1}$ is the real solution, and $s_{01,2}$ and $s_{01,3}$ are conjugated complex solutions. If $\Delta=0, A$ and $B$ are 
both real numbers with $A=B$. The three solutions are

$$
\begin{aligned}
& s_{01,1}=2 A^{1 / 3}+\Gamma, \\
& s_{01,2}=-A^{1 / 3}+\Gamma, \\
& s_{01,3}=-A^{1 / 3}+\Gamma,
\end{aligned}
$$

namely the three solutions are all real and $s_{01,2}=s_{01,3}$. If $\Delta<0, A$ and $B$ are conjugated complex numbers with $A^{1 / 3}=C+i D$ and $B^{1 / 3}=C-i D$. The three solutions are

$$
\begin{aligned}
& s_{01,1}=2 C+\Gamma, \\
& s_{01,2}=-C-\sqrt{3} D+\Gamma, \\
& s_{01,3}=-C+\sqrt{3} D+\Gamma,
\end{aligned}
$$

namely the three solutions are all real solutions.

Thus, $\Delta$ is the discriminant of the cubic nonlinear equation. According to Eq. (39), we have

$$
\frac{\Delta}{\Delta_{0}}=-4\left[\left(\varepsilon_{r}+1\right) k_{0} R-\sigma_{g, i}^{(1)} \eta_{0}\right]^{3}+27 k_{0}^{2} R^{2} \sigma_{g, i}^{(3)} \eta_{0}^{2} I_{0},
$$

where $\Delta_{0}=\pi^{6} k_{0}^{12} R^{12} /\left(6912 \sigma_{g, i}^{(3) 3} \eta_{0}^{6} I_{0}^{3}\right)$ is always a negative number. If the working frequency of bistable scattering is chosen as $f \geq f_{0}$, the first term in Eq. $49 p$ is negative and the determinant $\Delta$ is always positive. Thus Eq. (26) has only one real solution. Whereas, if $f<f_{0}$, the first term in Eq. 49 is positive and the determinate $\Delta$ may be positive or negative depending upon the value of $I_{0}$. Thus Eq. (26) has three real solutions for

$$
0<I_{0}<\frac{4\left[\left(\varepsilon_{r}+1\right) k_{0} R-\sigma_{g, i}^{(1)} \eta_{0}\right]^{3}}{27 k_{0}^{2} R^{2} \sigma_{g, i}^{(3)} \eta_{0}^{2}},
$$

two different real solutions for

$$
I_{0}=\frac{4\left[\left(\varepsilon_{r}+1\right) k_{0} R-\sigma_{g, i}^{(1)} \eta_{0}\right]^{3}}{27 k_{0}^{2} R^{2} \sigma_{g, i}^{(3)} \eta_{0}^{2}},
$$

and only one real solution for $I_{0}=0$ and

$$
I_{0}>\frac{4\left[\left(\varepsilon_{r}+1\right) k_{0} R-\sigma_{g, i}^{(1)} \eta_{0}\right]^{3}}{27 k_{0}^{2} R^{2} \sigma_{g, i}^{(3)} \eta_{0}^{2}} .
$$

According to the above discussion, the working frequency of bistable scattering should be smaller than the superscattering frequency (Supplement 1, Section 3). Figs. 3(a)-(b) show the scattering coefficients and NSCSs for different incident field intensities at $f=12.00 \mathrm{THz}$, where $\mu_{c}=0.35 \mathrm{eV}$ and $\tau=\infty$. Bistable scattering occurs under these parameters. For $I_{0}=0$, Eq. 26 has only one real solution, which corresponds to the starting points of the green curves. If $I_{0}$ increases from 0 , the second term in Eq. 49 increases. According to Eq. (50),
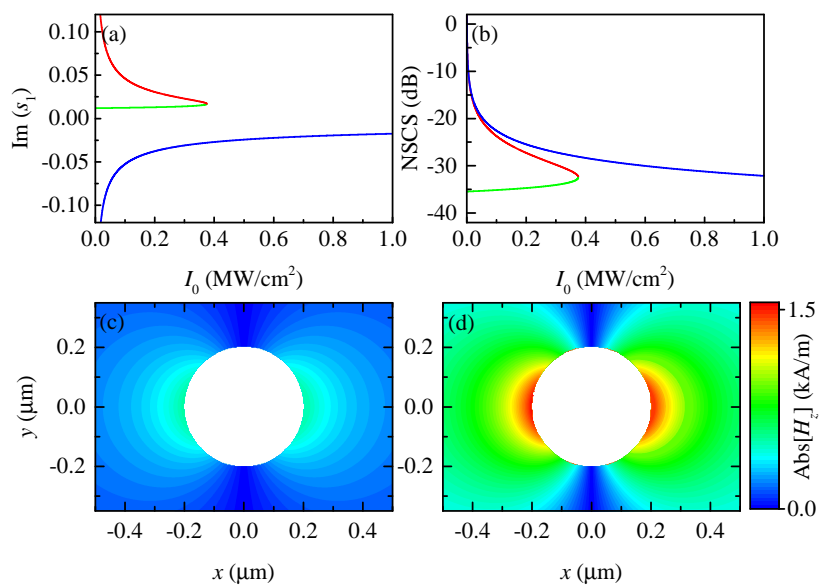

FIG. 3: (a) The scattering coefficients and (b) NSCSs for different incident field intensities. The curves with different colors correspond to different branches. (c)-(d) For $I_{0}=0.2$ $\mathrm{MW} / \mathrm{cm}^{2}$, the scattered fields which corresponds to the "off" and "on" states are shown, respectively, where the fields inside the dielectric nanowire are not shown. The parameters are $f=12.00 \mathrm{THz}, \mu_{c}=0.35 \mathrm{eV}$, and $\tau=\infty$.

if the incident field intensity is smaller than a threshold, one incident field intensity corresponds to three scattering coefficients, where two coefficients are positive and one coefficient is negative. Specially, if $I_{0}$ increases to the threshold where $\Delta=0$, the two positive scattering coefficients are equal. If $I_{0}$ continue increases, only the negative scattering coefficient still exists according to Eq. (52).

The fulfillment of $\Delta<0$ at a certain range of incident field intensity insures the existence of bistable scattering. As shown in Fig. 3(b), the bistable behavior can be used as a scattering switch. The bottom green curve and the top blue curve are two stable scattering states, and the middle red curve is an instable scattering state. Due to the instability, usually the scattering cannot exist on this state. The top and bottom stable states can be treated as "on" and "off" states of a scattering switch, respectively. For $I_{0}=0.2 \mathrm{MW} / \mathrm{cm}^{2}$, the scattered fields which corresponds to the "off" and "on" states are shown in Fig. 3(c)-(d), respectively, where the fields in the dielectric nanowire are not shown. The scattering switch has a high contrast between the fields. According to the analytical results, there are two intensity thresholds to control the switch. The scattering changes from "off" to "on" at the switching-up intensity $T_{\text {on }}$, and the scattering changes from "on" to "off" at the switching-down intensity $T_{\text {off. }}$ In this lossless nonlinear scattering model, $T_{\text {off }}=0$ and

$$
T_{\mathrm{on}}=\frac{4\left[\left(\varepsilon_{r}+1\right) k_{0} R-\sigma_{g, i}^{(1)} \eta_{0}\right]^{3}}{27 k_{0}^{2} R^{2} \sigma_{g, i}^{(3)} \eta_{0}^{2}} .
$$

The switching-up intensity $T_{\text {on }}$ is inversely dependent on the nonlinear surface conductivity of graphene, namely a 

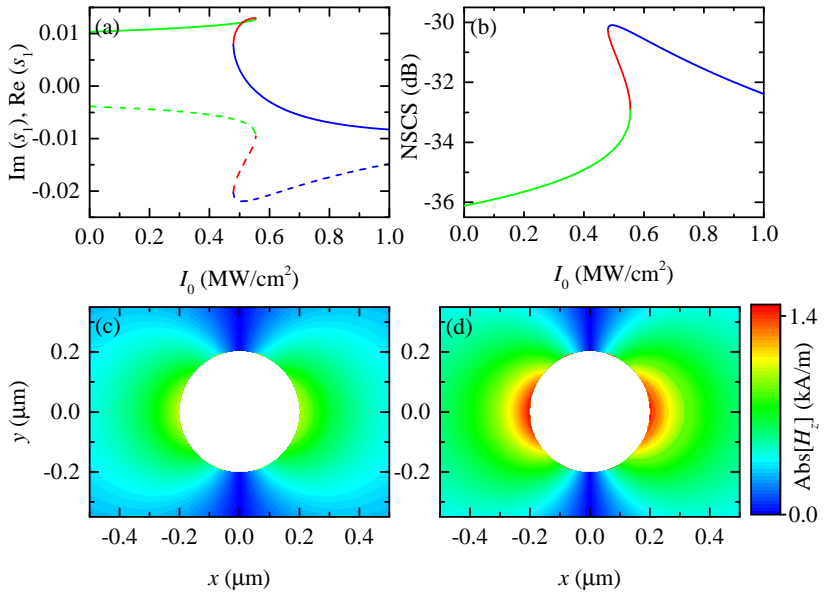

FIG. 4: (a) The scattering coefficients and (b) NSCSs for different incident field intensities. The curves with different colors correspond to different branches. In (a), the solid curve denotes $\operatorname{Im}\left(s_{1}\right)$, and the dashed curve denotes $\operatorname{Re}\left(s_{1}\right)$. (c)-(d) For $I_{0}=0.5 \mathrm{MW} / \mathrm{cm}^{2}$, the scattered fields which corresponds to the "off" and "on" states are shown, respectively, where the fields inside the dielectric nanowire are not shown. The parameters are $f=12.00 \mathrm{THz}, \mu_{c}=0.35 \mathrm{eV}$, and $\tau=0.30$ ps.

low intensity threshold requires a large $\sigma_{g, i}^{(3)}$.

\section{B. Lossy Case}

Next, we consider the lossy case, where the relaxation time of graphene is finite. In this case, Eq. (17) can be solved numerically (Supplement 1, Section 3). Figs. 4(a)(b) show the scattering coefficients and NSCSs for different incident field intensities at $f=12.00 \mathrm{THz}$, where $\mu_{c}=0.35 \mathrm{eV}$ and $\tau=0.30 \mathrm{ps}$. At this working frequency, both the scattering coefficient and NSCS show more interesting phenomena.

In Fig. 3(a) where $\tau=\infty$, the solid red and blue curves extend to the positive and negative infinities, respectively. In contrast, the solid red and blue curves in Fig. 4(a) are connected to each other at a certain incident field intensity, and a loop is formed by the three curves. Meanwhile, due to the finite relaxation time of graphene, the real part of the scattering coefficient is nonzero, and it demonstrates a bistable behavior with a dip, as shown by the dashed curves in Fig. 4(a).

Both the real and imaginary parts of the scattering coefficient contribute to the NSCS. Compared with the NSCS in Fig. 3(b), the NSCS in Fig. 4(b) has larger switching-up and switching-down intensities. Specially, the switching-down intensity is no longer equal to zero and the difference between the switching intensities defined as $\Delta T=T_{\text {on }}-T_{\text {off }}$ is decreased. Similarly, we also plot the scattered fields which corresponds to the "off" and "on" states in Fig. [4(c)-(d), respectively, where $I_{0}=0.5 \mathrm{MW} / \mathrm{cm}^{2}$, and the fields in the dielectric nanowire are not shown. The contrast between the fields is decreased due to the finite relaxation time of graphene. This implies that, the relaxation time of graphene plays a significant role in the bistable scattering.

Finally, we would like to note the validity our nonlinear scattering model. In Figs. 34 the ratio between the nonlinear and linear parts of the surface conductivity of graphene is less than $1 \times 10^{-10}$. This implies that the $\theta$ dependent nonlinear part only adds a small perturbation to the total surface conductivity and the field intensity along the graphene coating can be homogenized using the mean field approximation. Besides, in both the lossless and lossy cases, we use the approximations of $k_{0} R \ll 1$ and $k R \ll 1$, where the Bessel function and Hankel function are replaced by their respective asymptotic expansions in the nonlinear scattering model. By comparing the NSCSs with and without these approximations, we show that this kind of approximations is also valid (Supplement 1, Section 4). Our nonlinear scattering model provides a simple way to understand the bistable scattering.

\section{DISCUSSION}

According to the discussion in the previous section, the bistable scattering strongly dependents on the relaxation time of graphene, which describes the dissipation loss. Figs. 5(a)-(c) show the imaginary and real parts of the scattering coefficients and the NSCSs for different relaxation times, where $f=12.00 \mathrm{THz}, \mu_{c}=0.35 \mathrm{eV}$, and the values of $\tau$ are taken as $0.23 \mathrm{ps}, 0.30 \mathrm{ps}$, and $0.50 \mathrm{ps}$, respectively. The cases for $\tau=\infty$ are also plotted for comparison.

For $\tau=\infty$, two branches of the imaginary part of $s_{1}$ extend to the positive infinity and negative infinity, respectively. If $\tau$ decreases, the two branches connect to each other when the incident field intensity $I_{0}=T_{\text {off }}$, as shown in Fig. 5(a). A loop is formed by the three branches due to the existence of the switching-down intensity $T_{\text {off }}$ and the switching-up intensity $T_{\text {on }}$. However, if $\tau$ continues to decrease, the loop shrinks gradually. When the relaxation time is small enough, the loop disappears and there is no bistable behavior.

The real part of the scattering coefficient is emerged due to the finite relaxation time of graphene. For $\tau=\infty$, the real part of $s_{1}$ is always equal to zero. If $\tau$ decreases, two branches of the real part form a dip, as shown in Fig. 5(b). The three branches are connected to each other, and exhibit a bistable behavior. However, if $\tau$ continues to decrease, the dip becomes smooth gradually. When the relaxation time is small enough, the dip almost disappears and there is no bistable behavior.

The simultaneous existences of the loop from the imaginary part and the dip from the real part of the scattering coefficient indicate the existence of the bistable scattering. As shown in Fig. 5(c), for different relaxation times of graphene, the NSCSs show bistable scattering 

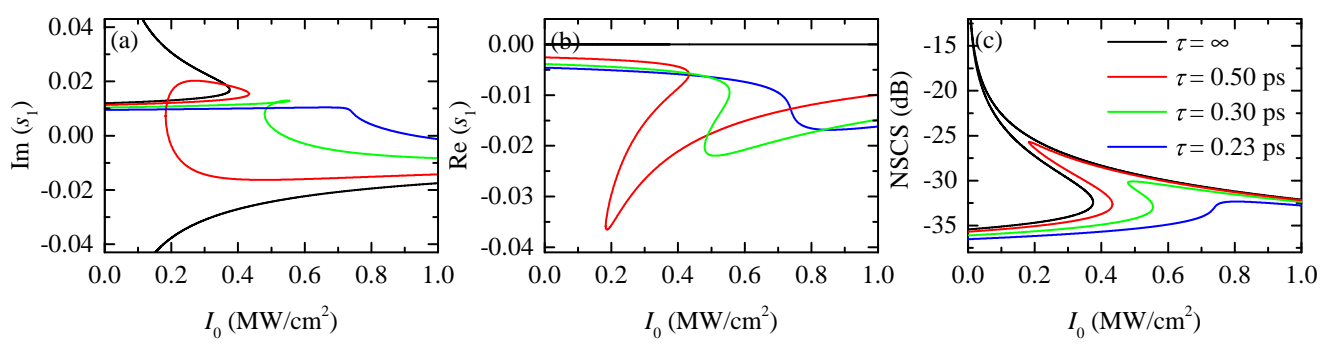

FIG. 5: (a) The imaginary and (b) real parts of the scattering coefficients and (c) the NSCSs for different relaxation times of graphene, where $f=12.00 \mathrm{THz}, \mu_{c}=0.35$, and the values of $\tau$ are taken as $0.23 \mathrm{ps}, 0.30 \mathrm{ps}$, and $0.50 \mathrm{ps}$, respectively. The cases for $\tau=\infty$ are also plotted for comparison
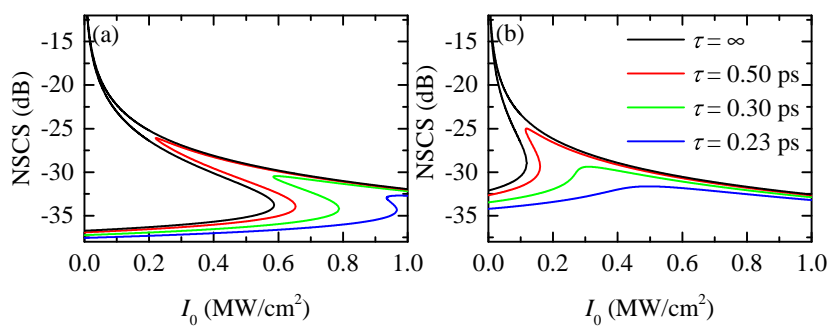

FIG. 6: The NSCSs for different relaxation times of graphene, where $\mu_{c}=0.35 \mathrm{eV}, f=11.90 \mathrm{THz}$ in (a) and $f=12.20 \mathrm{THz}$ in (b), and the values of $\tau$ are taken as $0.23 \mathrm{ps}, 0.30 \mathrm{ps}$, and $0.50 \mathrm{ps}$, respectively. The cases for $\tau=\infty$ are also plotted for comparison.

with different hysteresis curves. With the decreasing of $\tau$, the switching-up and switching-down intensities both decrease, and the difference between the switching intensities also decreases. Meanwhile, the maximum NSCS at the curve decreases sharply. If $\tau$ continues to decrease, the bistable scattering disappears and the hysteresis curve becomes a smooth curve gradually.

From Fig. 5, we can see that, if the incident frequency is fixed, in order to show the bistable scattering, the relaxation time of graphene should be large enough. From one hand, if the relaxation time is small, we need large switching-up and switching-down intensities, and the bistable scattering requires a large switching threshold. From another hand, if the relaxation time of graphene is too small, there is no bistable scattering at all. Thus, the working frequency and the performance of bistable scattering are restricted by the dissipation loss of graphene.

In order to further explain this restriction, in Fig. 6 we show the bistable scatterings at $f=11.90 \mathrm{THz}$ and $f=12.20 \mathrm{THz}$, respectively. At $f=11.90 \mathrm{THz}$, bistable scatterings are shown for all the three values of $\tau$. While at $f=12.20 \mathrm{THz}$, bistable scattering is only shown for $\tau=0.50 \mathrm{ps}$. In other words, at a low working frequency, bistable scattering occurs for a small relaxation time, and a large switching threshold is required. To decrease the switching threshold, the working frequency should be increased towards to the superscattering frequency. But if the working frequency is high, the bistable scattering is more sensitive to the relaxation time. To realize the bistable scattering at a high working frequency, the relaxation time of graphene must be large enough.

The chemical potential of graphene and the permittivity of the dielectric nanowire also affect the bistable scattering (Supplement 1, Section 5). According to Ref. 39], the changes of the chemical potential and the permittivity are equivalent with the change of the superscattering frequency. And the shift of the superscattering frequency is equivalent with the reverse shift of the working frequency. The performance of bistable scattering is still restricted by the relaxation time of graphene.

\section{CONCLUSION}

In conclusion, we have developed a nonlinear scattering model for a graphene-coated dielectric nanowire under the mean field approximation. The bistable scattering is discussed based on the semi-analytical solutions. We have found that the relaxation time of graphene is the main restriction to further decrease the switching threshold of bistable scattering, although the other parameters including the working frequency, the chemical potential of graphene, and the permittivity of the dielectric nanowire can be tuned freely. The development of grapheneboron nitride heterostructures that can support low-loss plasmons may shed new light on optical bistability in nonlinear graphene plasmonics [51, 52. Our work provides a theoretical guidance to further structure design and optimization, and application of optical bistability in the high speed all-optical signal processing.

\section{Funding Information}

National Natural Science Foundation of China (61625502, 61574127, 61601408, 61550110245); ZJNSF (LY17F010008); Fundamental Research Funds for the Central Universities (2016QNA5006); Top-Notch Young Talents Program of China; Innovation Joint Research Center for Cyber-Physical-Society System. 
See Supplement 1 for supporting content.

[1] H. M. Gibbs, Optical Bistability: Controlling Light with Light (Academic Press, Orlando, 1985).

[2] E. Abraham and S. D. Smith, Optical bistability and related devices, Rep. Prog. Phys. 45, 815 (1982).

[3] M. Soljačić, M. Ibanescu, S. G. Johnson, Y. Fink, and J. D. Joannopoulos, Optimal bistable switching in nonlinear photonic crystals, Phys. Rev. E 66, 055601(R) (2002).

[4] M. F. Yanik, S. Fan, and M. Soljačić, High-contrast alloptical bistable switching in photonic crystal microcavities, Appl. Phys. Lett. 83, 2739 (2003).

[5] C. Min, P. Wang, C. Chen, Y. Deng, Y. Lu, H. Ming, T. Ning, Y. Zhou, and G. Yang, All-optical switching in subwavelength metallic grating structure containing nonlinear optical materials, Opt. Lett. 33, 869 (2008).

[6] K. Okumura, Y. Ogawa, H. Ito, and H. Inaba, Optical Bistability and Monolithic Logic Functions Based on Bistable Laser/Light-Emitting Diodes, IEEE J. Quantum Elect. QE-21, 377 (1985).

[7] A. C. Walker, Application of bistable optical logic gate arrays to all-optical digital parallel processing, Appl. Opt. 25, 1578 (1986).

[8] W. Zhang, Y. Jiang, Y. Zhu, F. Wang, and Y. Rao, Alloptical bistable logic control based on coupled Tamm plasmons, Opt. Lett. 38, 4092 (2013).

[9] T. E.-Ortega and T. C. H. Liew, Complete architecture of integrated photonic circuits based on AND and NOT logic gates of exciton polaritons in semiconductor microcavities, Phys. Rev. B 87, 195305 (2013).

[10] D. A. B. Miller, S. Des Smith, and C. T. Seaton, Optical Bistability in Semiconductors, QE-17, 312 (1981).

[11] M. F. Yanik, S. Fan, M. Soljačić, and J. D. Joannopoulos, All-optical transistor action with bistable switching in a photonic crystal cross-waveguide geometry, Opt. Lett. 28, 2506 (2003).

[12] D. Ballarini, M. De Giorgi, E. Cancellieri, R. Houdré, E. Giacobino, R. Cingolani, A. Bramati, G. Gigli, and D. Sanvitto, All-optical polariton transistor, Nat. Commun. 4, 1778 (2013).

[13] W. J. Firth, Optical Memory and Spatial Chaos, Phys. Rev. Lett. 61, 329 (1988).

[14] T. Tanabe, M. Notomi, S. Mitsugi, A. Shinya, and E. Kuramochi, Fast bistable all-optical switch and memory on a silicon photonic crystal on-chip, Opt. Lett. 30, 2575 (2005).

[15] L. Liu, R. Kumar, K. Huybrechts, T. Spuesens, G. Roelkens, E.-J. Geluk, T. de Vries, P. Regreny, D. V. Thourhout, R. Baets, and G. Morthier, An ultra-small, low-power, all-optical flip-flop memory on a silicon chip, Nat. Photon. 4, 182 (2010).

[16] K. Nozaki, A. Shinya, S. Matsuo, Y. Suzaki, T. Segawa, T. Sato, Y. Kawaguchi, R. Takahashi, and M. Notomi, Ultralow-power all-optical RAM based on nanocavities, Nat. Photon. 6, 248 (2012).

[17] W. L. Barnes, A. Dereux, and T. W. Ebbesen, Surface plasmon subwavelength optics, Nature 424, 824 (2003).

[18] J. A. Schuller, E. S. Barnard, W. Cai, Y. C. Jun, J. S. White, and M. I. Brongersma, Plasmonics for extreme light concentration and manipulation, Nat. Mat. 9, 193
(2010).

[19] M. Kauranen and A. V. Zayats, Nonlinear plasmonics, Nat. Photon. 7, 737 (2012).

[20] G. A. Wurtz, R. Pollard, and A.V. Zayats, Optical Bistability in Nonlinear Surface-Plasmon Polaritonic Crystals, Phys. Rev. Lett. 97, 057402 (2006).

[21] C. Min, P. Wang, X. Jiao, Y. Deng, and H. Ming, Optical bistability in subwavelength metallic grating coated by nonlinear material, Opt. Exp. 15, 12368 (2007).

[22] F. Zhou, Y. Liu, Z.-Y. Li, and Y. Xia, Analytical model for optical bistability in nonlinear metal nano-antennae involving Kerr materials, Opt. Exp. 18, 13337 (2010).

[23] C. Argyropoulos, P.-Y. Chen, F. Monticone, G. D'Aguanno, and A. Alù, Nonlinear Plasmonic Cloaks to Realize Giant All-Optical Scattering Switching, Phys. Rev. Lett. 108, 263905 (2012).

[24] H. Lu and X. M. Liu, Optical bistability in subwavelength compound metallic grating, Opt. Exp. 21, 13794 (2013).

[25] C. Argyropoulos, C. Ciracì, and David R. Smith, Enhanced optical bistability with film-coupled plasmonic nanocubes, Appl. Phys. Lett. 104, 063108 (2014).

[26] H. Chen, Y. Zhang, B. Zhang, and Lei Gao, Optical bistability in a nonlinear-shell-coated metallic nanoparticle, Sci. Rep. 6, 21741 (2016).

[27] W. J. Yu, H. Sun, and L. Gao, Optical bistability in coreshell magnetoplasmonic nanoparticles with magnetocontrollability, Opt. Exp. 24, 22272 (2016).

[28] M. M. Glazov, S. D. Ganichev, High frequency electric field induced nonlinear effects in graphene, Phys. Rep. 535, 101 (2014).

[29] E. Hendry, P. J. Hale, J. Moger, and A. K. Savchenko, Coherent Nonlinear Optical Response of Graphene, Phys. Rev. Lett. 105, 097401 (2010).

[30] N. M. R. Peres, Yu. V. Bludov, J. E. Santos, A.-P. Jauho, and M. I. Vasilevskiy, Optical bistability of graphene in the terahertz range, Phys. Rev. B 90, 125425 (2014).

[31] X. Dai, L. Jiang, and Y. Xiang, Tunable optical bistability of dielectric/nonlinear graphene/dielectric heterostructures, Opt. Exp. 23, 6479 (2015).

[32] M. A. Sharif, M. H. Majles Ara, B. Ghafary, S. Salmani, S. Mohajer, Experimental observation of low threshold optical bistability in exfoliated graphene with low oxidation degree, Opt. Mater. 53, 80 (2016).

[33] X. Dai, L. Jiang, and Y. Xiang, Low threshold optical bistability at terahertz frequencies with graphene surface plasmons, Sci. Rep. 5, 12271 (2015).

[34] H. Wang, J. Wu, J. Guo, L. Jiang, Y. Xiang, and S. Wen, Low-threshold optical bistability with multilayer graphene-covering Otto configuration, J. Phys. D 49, 255306 (2016).

[35] T. Christensen, W. Yan, A.-P. Jauho, M. Wubs, and N. A. Mortensen, Kerr nonlinearity and plasmonic bistability in graphene nanoribbons, Phys. Rev. B 92, 121407(R) (2015).

[36] Q. Bao, J. Chen, Y. Xiang, K. Zhang, S. Li, X. Jiang, Q.-H. Xu, K. P. Loh, and T. Venkatesan, Graphene Nanobubbles: A New Optical Nonlinear Material, Adv. Opt. Mater. 3, 744 (2015). 
[37] P.-Y. Chen, and A. Alù, Atomically Thin Surface Cloak Using Graphene Monolayers, ACS Nano, 5, 5855 (2011).

[38] M. Farhat, C. Rockstuh, and H. Bağci, A 3D tunable and multi-frequency graphene plasmonic cloak, Opt. Exp. 21, 12592 (2013).

[39] R. Li, X. Lin, S. Lin, X. Liu, and H. Chen, Tunable deepsubwavelength superscattering using graphene monolayers, Opt. Lett. 40, 1651 (2015).

[40] R. Li, X. Lin, S. Lin, X. Liu, and H. Chen, Atomically thin spherical shell-shaped superscatterers based on a Bohr model, Nanotechnology 26, 505201 (2015).

[41] R. Li, B. Zheng, X. Lin, R. Hao, S. Lin, W. Yin, E. Li, and H. Chen, Design of Ultracompact Graphene-Based Superscatterers, IEEE J. Sel. Top. Quant. 23, 4600208 (2017).

[42] Y. Gao, G. Ren, B. Zhu, J. Wang, and S. Jian, Singlemode graphene-coated nanowire plasmonic waveguide, Opt. Lett. 39. 5909 (2014).

[43] Y. Gao, G. Ren, B. Zhu, H. Liu, Y. Lian, and S. Jian, Analytical model for plasmon modes in graphene-coated nanowire, Opt. Exp. 22, 24322 (2014).

[44] A. V. Gorbach, A. Marini, and D. V. Skryabin, Graphene-clad tapered fiber: effective nonlinearity and propagation losses, Opt. Lett. 38, 5244 (2013).

[45] R. Li, X. Lin, S. Lin, X. Zhang, E. Li, and H. Chen, Graphene induced mode bifurcation at low input power,
Carbon 98, 463 (2016).

[46] T. Christensen, A.-P. Jauho, M. Wubs, and N. A. Mortensen, Localized plasmons in graphene-coated nanospheres, 91, 125414 (2015).

[47] D. A. Smirnova, I. V. Shadrivov, A. E. Miroshnichenko, A. I. Smirnov, and Y. S. Kivshar, Second-harmonic generation by a graphene nanoparticle, Phys. Rev. B 90, 035412 (2014).

[48] Y. Huang, A. E. Miroshnichenko, and L. Gao, Lowthreshold optical bistability of graphene-wrapped dielectric composite, Sci. Rep. 6, 23354 (2016).

[49] A. Ishimaru, Electromagnetic Wave Propagation, Radiation, and Scattering (Prentice-Hall, New Jersey, 1991).

[50] M. Abramowitz and I. A. Stegun, Handbook of Mathematical Functions with Formulas, Graphs, and Mathematical Tables (Dover, 1965).

[51] C. R. Dean, A. F. Young, I. Meric, C. Lee, L. Wang, S. Sorgenfrei, K. Watanabe, T. Taniguchi, P. Kim, K. L. Shepard, and J. Hone, Boron nitride substrates for highquality graphene electronics, 5, 722 (2010).

[52] A. Woessner, M. B. Lundeberg, Y. Gao, A. Principi, P. A.-González, M. Carrega, K. Watanabe, T. Taniguchi, G. Vignale, M. Polini, J. Hone, R. Hillenbrand, and F. H. L. Koppens, Highly confined low-loss plasmons in grapheneboron nitride heterostructures, 14, 421 (2015). 